\title{
More on fecal occult blood test misuse
}

\author{
Clarence Wong MD FRCPC ${ }^{1}$, Catherine Dubé MD MSc FRCPC ${ }^{2}$
}

$\mathrm{T}$ he current issue of the Journal includes a study by Narula et al (1) (pages 421-426) that nicely demonstrates the numerous reasons why fecal occult blood test (FOBT) use outside of screening purposes should be considered inappropriate and how it may, in fact, negatively impact patient care. The authors performed a chart review of all FOBTs ordered in Hamilton Health Sciences' (Hamilton, Ontario) acute care campuses over a three-month period in 2011, combined with a survey of health care providers on their practices regarding the use of FOBT. Use of point-of-care FOBT was not included. The clinical presentations were anemia, overt or suspected gastrointestinal (GI) bleeding, diarrhea, iron deficiency and dyspepsia. Thirty-four percent of patients underwent $\geq 2$ FOBTs, and the majority of tested patients were either on medications or on a diet that could interfere with the test. Only $50 \%$ of the FOBT positives were ever referred for a GI consultation and, most importantly, $27 \%$ of patients presenting with overt GI bleeding in whom an FOBT was ordered experienced a delay in the GI referral because of the FOBT process. The survey included 67 health care professionals (mainly primary care physicians and nurses); the most common reasons for ordering an FOBT were: symptoms potentially consistent with GI bleeding (84\%); anemia $(53 \%)$; iron deficiency with or without anemia $(31 \%)$; overt GI blood loss $(26 \%)$; and nonbloody diarrhea (10\%). Interestingly, screening for colorectal cancer was a cited reason in only $25 \%$ of the cases.

The authors highlight several important problems. Even if the use of the test was intially inappropriate, only one-half of those who tested positive were ever referred to the GI service, indicating an underlying indecisiveness of the ordering team about the overall management of the patient. In situations of uncertainty regarding the management of patients presenting with GI symptoms, these results would suggest that the mere act of ordering an FOBT provides a sense that something is being done anyway. The study by Narula et al (1) supports the evidence that FOBT use outside of screening purposes is actually harmful to patients. The fundamental problem with ordering an FOBT for any of the reasons listed by the survey respondents, outside of screening asymptomatic average-risk individuals, is that any of these presentations require workup independently of the FOBT result.

The results were similar to those reported in a retrospective review of FOBT use in 15 hospitals during 2005 in the Netherlands (2), where it was also shown that FOBT misuse creates a diagnostic delay. Both studies support the same simple conclusion: FOBT should not be used for purposes other than screening, and patients presenting with symptoms suggestive of an underlying malignancy or iron deficiency anemia require endoscopic evaluations.

In contrast, a meta-analysis examining the use of diagnostic tests to help identify individuals who are most likely to have an underlying malignancy among patients presenting with nonacute lower abdominal symptoms $(3,4)$ concluded that the use of FOBT and the fecal immunochemical test (FIT) in such patients had high sensitivity and specificity. There were, however, several concerns regarding the generalizability of the studies included in the meta-analysis in addition to significant heterogeneity that could not be explained.
Most importantly, as demonstrated in the study by Narula et al (1), there is a tendency for the FOBT to be used in individuals who require the referral anyway, and that a negative result would prevent a patient with concerning symptoms to access timely endoscopic investigations.

The problem of FOBT misuse goes beyond the care of individual patients. Colorectal cancer screening programs that rely on primary care physicians to initiate screening or distribute FOBT kits are also faced with problems of kit distribution to individuals who are not eligible for screening. Alternatively, requests for screening tends to occur once patients present with symptoms rather than being performed in asymptomatic patients. These situations result in two major problems: suboptimal screening participation, and increased costs and resource use for individuals who are outside the target population.

This problem is compounded by the fact that most screening programs in Canada are now using the FIT. In Alberta, guaiac FOBT was replaced with quantitative FIT in November 2013 and is since no longer available in community laboratories. A campaign that included a provincial update on colorectal cancer guidelines was initiated to review FIT indications. On review of the first six months of FIT usage, the Alberta Colorectal Cancer Screening Program found that of the 150,000 kits completed to date, $>13 \%$ were in individuals $<40$ or $>75$ years of age (ie, outside the screening guidelines) (5). While the exact indication for these kits is largely unknown, it is likely that an unacceptably high number was completed for diagnostic purposes. This increases wait times for colonoscopy and delays appropriate assessment, even for individuals with appropriate indications. Of the indications for FIT it was able to review, the Alberta Colorectal Cancer Screening Program identified three common types of FIT misuse: diagnostic-based indications, as described by Narula et al (1); point-of-care assessments; and screening outside of recommended age ranges, as described above. Examples of inappropriate use included the assessment of rectal bleeding in children with FIT, use of FIT in emergency units and surveillance of patients with a history of colorectal cancer with FIT instead of colonoscopy. Even in cases in which FIT was used for screening, FIT kits were sometimes given to patients with significant multiple morbidities or who were $>90$ years of age. A campaign supported by the Canadian Medical Association to guide discussion for patients and physicians on inappropriate diagnostic tests has been initiated through 'Choosing Wisely Canada'. One of the current recommendations includes "Avoid colorectal cancer screening tests on asymptomatic patients with a life expectancy of less than 10 years and no family or personal history of colorectal neoplasia" (6).

In summary, the Journal presents yet another study demonstrating multiple ways by which FOBT is misused and how this can negatively impact access for patients with clear indications for further evaluations. Such situations would be best avoided if the FOBT was simply not available for use outside of screening purposes. Based on this report, this would not only be a cost-saving intervention but also improve the quality of patient care.

${ }^{1}$ Divison of Gastroenterology, Royal Alexandra Hospital, Edmonton, Alberta; ${ }^{2}$ Division of Gastroenterology, The Ottawa Hospital, Ottawa, Ontario

Correspondence: Dr Catherine Dubé, Department of Medicine, Division of Gastroenterology, University of Ottawa, Room A194, The Ottawa

Hospital - Civic Campus, 1053 Carling Avenue, Ottawa, Ontario K1Y 4E9. E-mail cdube@toh.on.ca

Received and accepted for publication August 7, 2014 


\section{Editorial}

\section{REFERENCES}

1. Narula N, Ulic D, Al-Dabbagh RA, et al. Fecal occult blood testing as a diagnostic test in symptomatic patients is not useful: A retrospective chart review. Can J Gastroenterol Hepatol 2014;28:421-6.

2. Van Rijn AF, Stroobants AK, Deutecom M, et al. Inappropriate use of the faecal occult blood test in a university hospital in the Netherlands. Eur J Gastroenterol Hepatol 2012;24:1266-9.

3. Jellema P, van der Windt DAWM, Bruinvels DJ, et al. Value of symptoms and additional diagnostic tests for colorectal cancer in primary care: Systematic review and meta-analysis.

BMJ 2010;340:c1269.
4. Del Giudice L, Vella E, Hey A, et al. Referral of patients with suspected colorectal cancer by family physicians and other primary care providers: Evidentiary base. Toronto: Cancer Care Ontario; 2011 April 24. Program in Evidence-Based Care Evidence-Based Series 24-1: Section 2.

5. Alberta Colorectal Cancer Screening Program, Alberta Health Services. 2014.

6. Choosing Wisely Canada. Canadian Association of General Surgeons. Six Things Physicians and Patients Should Question $<$ www.choosingwiselycanada.org/recommendations/canadianassociation-of-general-surgeons-3/> (Accessed August 1, 2014). 


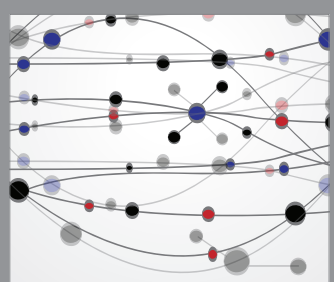

The Scientific World Journal
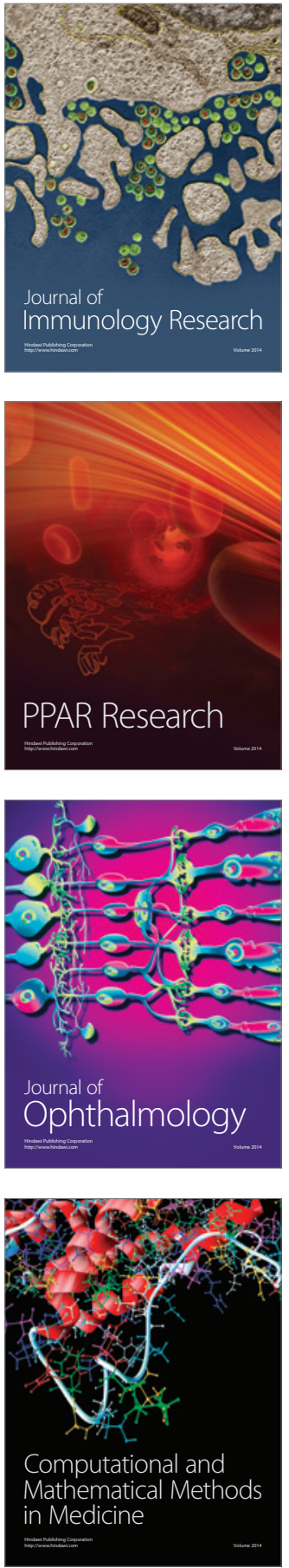

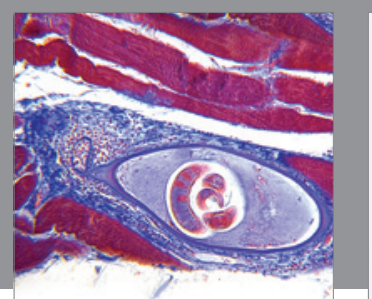

Gastroenterology Research and Practice

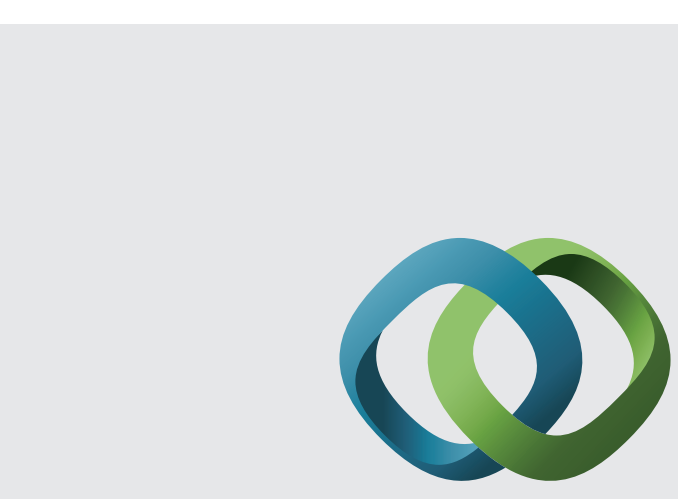

\section{Hindawi}

Submit your manuscripts at

http://www.hindawi.com
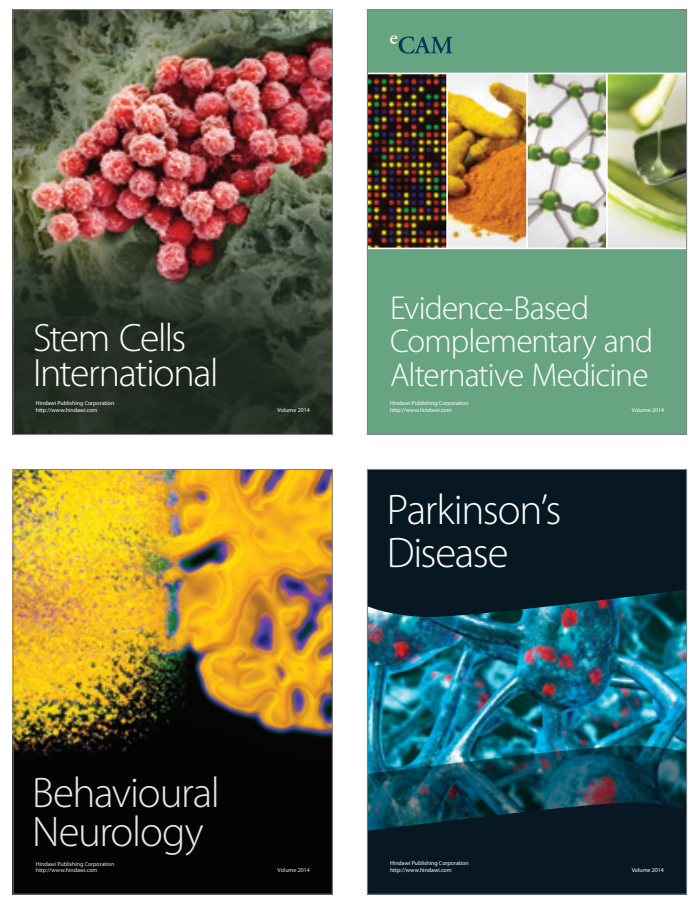
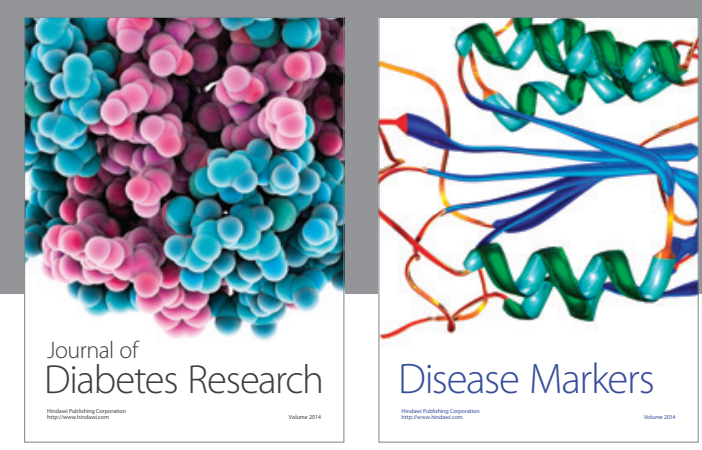

Disease Markers
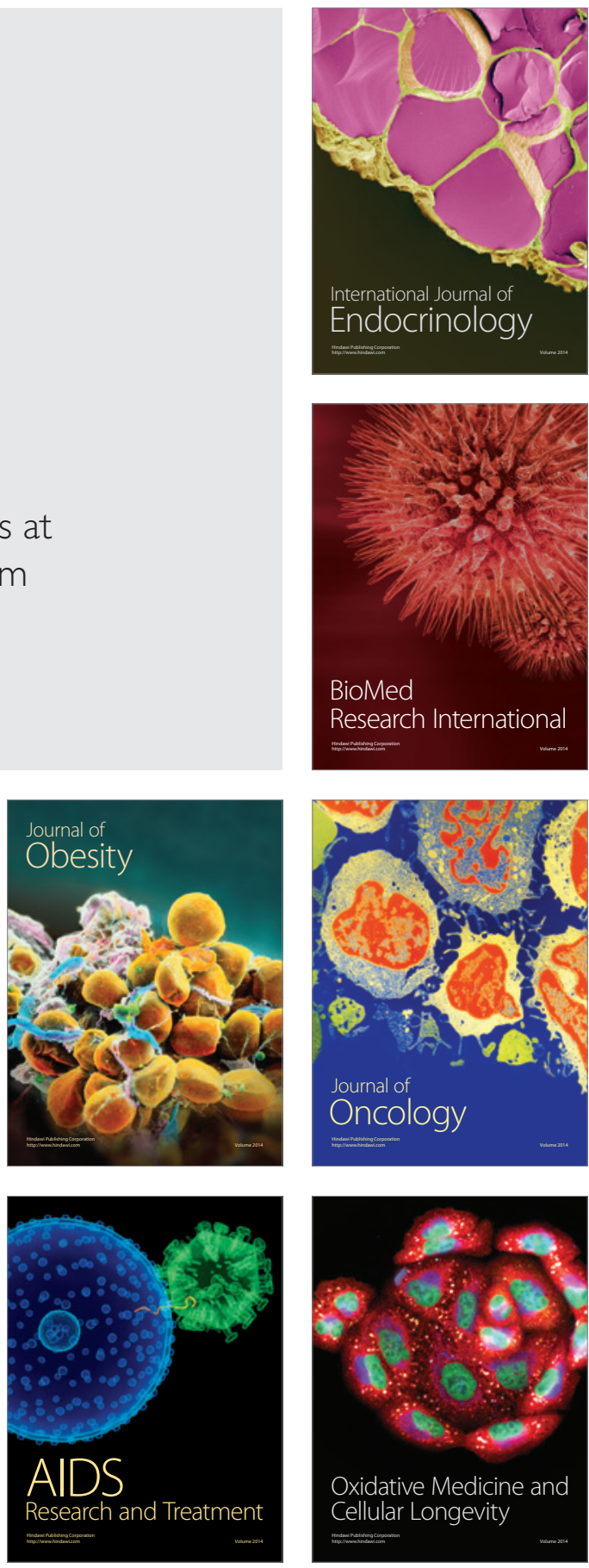